\title{
Polarization-sensitive optical phenomena in semiconducting and metallic nanowires
}

\author{
H. E. Ruda and A. Shik \\ Centre for Advanced Nanotechnology, University of Toronto, Toronto M5S 3E4, Canada \\ (Received 3 June 2005; revised manuscript received 26 July 2005; published 8 September 2005)
}

\begin{abstract}
An analysis is presented of optical absorption and emission in semiconducting, metallic, and mixed coreshell nanowires are considered theoretically. Due to image forces caused by the difference in dielectric constants between nanowires and their environment, absorption and emission coefficients are different for light polarized parallel or perpendicular to the nanowire axis. As a result, the intensity and spectra of absorption, luminescence, luminescence excitation, and photoconductivity in nanowires or arrays of parallel nanowires are strongly polarization-sensitive. In random nanowire arrays, the effect may result in a "polarization memory" where the polarization of the luminescence is determined by the polarization of the exciting light. In lightemitting core-shell nanowire structures, the described redistribution of ac electric field may result in an enhancement of the luminescence from the nanowire cores in the frequency region corresponding to the plasmon resonances of their metallic shells.
\end{abstract}

DOI: $10.1103 /$ PhysRevB.72.115308

PACS number(s): 78.67.-n, 71.45.Gm

\section{INTRODUCTION}

This paper is devoted to optical phenomena in semiconducting nanowires having a dielectric constant $\varepsilon$ essentially from that of their environment, when polarization effects (image forces) play an important role. These effects are insignificant in heterojunction-based nanowire structures where the nanowire material (e.g., GaAs) is surrounded by a wider-gap material (e.g., AlGaAs) having a close value of $\varepsilon$. However, in the recent years, a large number of works have emerged devoted to fabrication and investigation of semiconductor nanowires grown either in a free-standing fashion from catalyst particle nanoislands (see, e.g., Ref. 1) or in a dielectric template with cylindrical pores. ${ }^{2}$ Since the dielectric constants of semiconductors $\varepsilon$ usually exceed 10 , this situation corresponds to a nanowire with a high $\varepsilon$ embedded in a medium (vacuum or dielectric) with lower dielectric constant $\varepsilon_{0}$. This situation will also apply if the nanowires are separated from the substrate and dissolved in some nonpolar liquid, which is often done for optical measurements. Such effects of electrostatic mismatch (image forces) can be observed not only semiconducting but also for metallic nanowires, where $\varepsilon$ also differs noticeably from $\varepsilon_{0}$ and, besides, has a complex character and frequency dispersion.

The role of image forces in electrostatic effects in nanowires was studied in the following: ${ }^{3}$ this includes considerations of modification of the Coulomb potential and binding energy of excitons and impurity centers. In this paper, we consider the influence of these phenomena on the intensity of the optically-induced electric field inside nanowires, which, in turn, determines all light-induced phenomena, such as optical absorption, luminescence, and photoconductivity. We show that even in relatively thick nanowires, where size effects are not significant and energy spectrum, as well as transport coefficeints, are isotropic, the difference between $\varepsilon$ and $\varepsilon_{0}$ results in a strong dependence of the abovementioned phenomena on the polarization of the exciting light. Similarly, light emitted by such nanowires appears to be strongly polarized, even if the probability of the corresponding optical transitions is independent of the orientation of the effective dipole moment. The results are also generalized to the case of core-shell nanowires containing several layers with different dielectric constants.

\section{UNIFORM NANOWIRES}

\section{A. Polarization of absorption}

If a cylindrical wire is placed in external electric field $\mathbf{E}_{0}$, its parallel component, $E_{\|}$, being continuous at the interface, remains the same inside a wire,

$$
E_{\|}=E_{0 \|} \cdot
$$

At the same time, the component normal to the wire axis, $E_{\perp}$, will be essentially suppressed inside the wire ${ }^{4}$

$$
E_{\perp}=\frac{2 \varepsilon_{0}}{\varepsilon+\varepsilon_{0}} E_{0 \perp}
$$

Though Eq. (2) was initially derived for static electric fields, it remains valid for high frequency fields as well (with $\varepsilon$ and $\varepsilon_{0}$ corresponding to the field frequency $\omega$ ) as long as the nanowire radius $a$ remains much less than the electromagnetic wavelength. For nanowires with $a<100 \mathrm{~nm}$ this covers microwave, infrared, and the whole visible light spectrum.

For $\varepsilon>\varepsilon_{0}$, Eq. (2) has two important consequences:

(1) The amplitude of the high-frequency electric field and, hence, the probability of optical transitions in a nanowire depend dramatically on the light polarization, acquiring maximal values for light with polarization parallel to the nanowire axis;

(2) illuminating with unpolarized light creates a highfrequency electric field in the nanowire with the predominat direction of polarization parallel to the nanowire axis.

The practical consequence of the first effect is a strong dependence of the absorption coefficient $k$ (both inter- and 
intraband) on the light polarization for a system of parallel wires. The ratio of $k$ for the two light polarizations is

$$
\frac{k_{\|}}{k_{\perp}}=\left|\frac{\varepsilon+\varepsilon_{0}}{2 \varepsilon_{0}}\right|^{2}
$$

For most semiconductors this factor exceeds 30 for free standing nanowires $\left(\varepsilon_{0}=1\right)$ and 4.7 for nanowires in $\mathrm{Al}_{2} \mathrm{O}_{3}$ matrix $\left(\varepsilon_{0} \simeq 3\right.$ in the optical spectral region $\left.{ }^{5}\right)$.

Note that so far we have considered classical electrodynamic effects caused exclusively by the difference of the refractive indices for wires and environment. In thin nanowires, due to size quantization, the optical matrix element becomes anisotropic, ${ }^{6,7}$ which may modify the described polarization effects so that Eq. (3) acquires an additional factor equal to the square of the ratio of the matrix elements for the two perpendicular directions.

Direct measurement of optical absorption in a nanowire system is difficult but it can be replaced by measurements of photoluminescence excitation or photoconductivity (for nanowires provided with contacts). All the effects discussed should be polarization-sensitive acquiring their maximum value for the exciting light polarization parallel to the nanowires. Such type of luminescence experiments with variable polarization of excitation in a system of parallel nanowires was described in Refs. 8-10. However, the object of these investigations was nanowire arrays in the GaAs/AlAs heterosystem with a weak difference between $\varepsilon$ and $\varepsilon_{0}$ and very thin (several nanometers), size-quantized nanowires. As a result, the observed angular polarization dependence was interpreted as caused by the above-mentioned anisotropy of the optical matrix element.

The discussed effects in their pure form were first observed and adequately interpreted in Ref. 11. The authors observed photoluminescence and photoconductivity in InP nanowires increasing up to 49 times when the polarization of the exciting light changed from perpendicular to parallel. This number exactly corresponds to Eq. (3) for InP in air. Similar effects in photoluminescence with a slightly lesser degree of polarization (maybe, owing to the misorientation of the nanowires discussed below) were also observed in $\mathrm{Si}$ nanowires. ${ }^{12}$

Contrary to nanowire arrays obtained by submicron lithography or grown in templates, free-standing nanowires are never strictly parallel. In this case, polarization measurements can be used to determine the angular distribution of the wire orientation. To treat this problem analytically, we choose the system of spherical angles $(\theta, \alpha)$ with $\theta$ measured from the mean direction of nanowires. If we consider an arbitrary electric field $\mathbf{E}_{0}=\left(E_{0}, \theta, \alpha\right)$ and some particular nanowire with orientation $\left(\theta^{\prime}, \alpha^{\prime}\right)$, then the angle between them $\gamma\left(\theta, \theta^{\prime}, \alpha, \alpha^{\prime}\right)=\cos ^{-1}\left(\cos \theta \cos \theta^{\prime}\right.$ $\left.+\sin \theta \sin \theta^{\prime} \cos \left(\alpha-\alpha^{\prime}\right)\right)$. In this case, the mean value of a squared projection of $\mathbf{E}_{0}$ to the nanowire axes $\overline{E_{0 \|}^{2}}$ $=\overline{E_{0}^{2}} \iint\left[\cos \gamma\left(\theta, \theta^{\prime}, \alpha, \alpha^{\prime}\right)\right]^{2} f\left(\theta^{\prime}, \alpha^{\prime}\right) \sin \theta^{\prime} d \theta^{\prime} d \alpha^{\prime}$, where $f$ is the distribution function of nanowire orientations. For simplicity, we characterize them by a uniform distribution with a width $\Delta: f(\theta, \alpha)=[2 \pi(1-\cos \Delta)]^{-1}$ at $\theta<\Delta$ and $f(\theta, \alpha)=0$ elsewhere. This gives

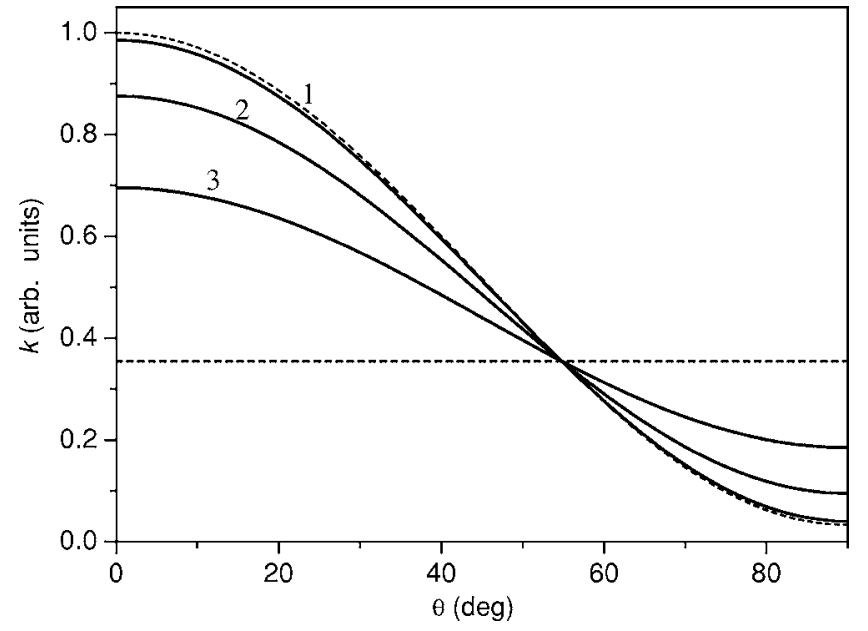

FIG. 1. Angular dependence of the absorption coefficient for nanowires with $\varepsilon=10$ in air at different orientation dispersion: $1-\Delta=10^{\circ} ; 2-\Delta=30^{\circ} ; 3-\Delta=50^{\circ}$. Dashed lines show the limiting cases of ideally ordered $(\Delta=0)$ and completely randomized nanowires.

$$
\begin{aligned}
\overline{E_{0 \|}^{2}}= & \frac{E_{0}^{2}}{1-\cos \Delta}\left[\frac{1}{3}-\frac{1}{4} \cos \Delta\left(1+\frac{1}{2} \sin ^{2} \theta\right)\right. \\
& \left.-\frac{1}{12} \cos 3 \Delta\left(1-\frac{3}{2} \sin ^{2} \theta\right)\right] .
\end{aligned}
$$

With $\Delta$ increasing from 0 to $\pi, \overline{E_{0 \|}^{2}}$ evolves from $E_{0}^{2} \cos ^{2} \theta$ to the isotropic value of $E_{0}^{2} / 3$ corresponding to complete randomization of orientation. Using Eqs. (1), (2), and (4), we obtain the average square of electric field strength inside a wire

$$
\overline{E^{2}}=\overline{E_{0 \|}^{2}}+\left(\frac{2 \varepsilon_{0}}{\varepsilon+\varepsilon_{0}}\right)^{2}\left(E_{0}^{2}-\overline{E_{0 \|}^{2}}\right) .
$$

Figure 1 shows the dependence of the absorbtion coefficient $k$ on the light polarization, which is proportional to the angular dependence of $\left(\overline{E^{2}} / E_{0}^{2}\right)$ for a semiconductor nanowire in air $\left(\varepsilon=10, \varepsilon_{0}=1\right)$ for several different dispersions of nanowire orientation $\Delta$. These characteristics must be compared with the dependence of wire luminescence on the polarization of exciting light. The point of maximal intensity gives the mean direction of wires while the amplitude of angular dependence for the known $\varepsilon$ and $\varepsilon_{0}$ allows one to estimate $\Delta$. In real experiments such an approach is correct if the standard deviation of light polarization $\delta$ is much less than $\Delta$, otherwise the experimentally determined $\Delta$ would be actually the sum of real $\Delta$ and $\delta$.

\section{B. Plasmon phenomena in metallic nanowires}

The most important parameter determining the abovementioned effects, is the nanowire dielectric constant $\varepsilon$ (more exactly, its ratio to that of its environment $\varepsilon_{0}$ ). Generally speaking, due to the frequency dispersion of $\varepsilon$, the optical anisotropy may depend on the light frequency $\omega$. However, in semiconductors this fact is of a minor importance. 
Even near the interband absorption edge, when $k$ changes by several orders of magnitude, $|\varepsilon|$ and, hence, the distribution of electric field and the ratio $k_{\|} / k_{\perp}$ [Eq. (3)] remain almost unchanged. On the contrary, in metals the frequency dependence $\varepsilon(\omega)$ may be very strong, and all the effects considered here acquire specific dependencies on the light frequency $\omega$ caused by plasmon-related phenomena. To analyze these theoretically, we use for $\varepsilon$ the simplest Drude expression

$$
\varepsilon=1-\frac{\omega_{p}^{2}}{\omega(\omega-i \nu)}
$$

where $\omega_{p}$ is the plasma frequency and $\nu$ is the scattering rate. Such an approach, fully applicable for simple, freeelectronlike metals, such as $\mathrm{Na}$, is also often used for metals with a complex Fermi surface by including in $\varepsilon$ some constant term describing the polarizability of $d$-electrons (see, e.g., Ref. 13), which results in renormalization of $\omega_{p}$ making it 2-3 times lower than the free-electron value. As to $\nu$ in nanostructures, it can exceed the corresponding value in a bulk metal due to additional interface scattering. That is why $\omega_{p}$ and $\nu$ may not quantitatively coincide with those for free electrons in bulk materials but rather should be considered as phenomenological parameters to be found (if necessary) from the best agreement with experiments.

It can be seen from Eq. (2) that at $\omega \rightarrow \omega_{p} / \sqrt{\varepsilon_{0}+1}$ the field component $E_{\perp}$ inside nanowires increases dramatically, being restricted only by the nonzero scattering rate of carriers, which, in turn, results in a noticeable absorption in this spectral region. This is the manifestation of well known plasmon effects in nanostructures ${ }^{14}$ which in our extremely anisotropic case, occur only for light polarized perpendicular to the nanowire axis. As a result, anisotropy of optical absorption given by Eq. (3), in metallic nanowires has an interesting spectral dependence. Far below the plasmon frequency (which in most metals corresponds to infrared radiation and the red part of visible spectrum), $|\varepsilon(\omega)|$ is very large, and the situation is similar to that in semiconductor nanowires where absorption is much higher for the light with parallel polarization. However, near the plasmon frequency absorption of the perpenducularly polarized light increases dramatically, changing the sign of anisotropy, as illustrated by Fig. 2. At $\omega \rightarrow \infty$ anisotropy tends to a constant value determined by $\varepsilon_{0}$ and equal to zero at $\varepsilon_{0}=1$.

Thus, the situation near the plasmon resonance is, to some extent, opposite to that of Sec. II A, where, due to large $\varepsilon$ of nanowire, electric field of light is polarized mostly along the wire axis. In metallic nanowires, $\varepsilon$ for light polarized perpendicular to the wires and having a frequency in the plasmon region is close to zero. For this reason, the electric field in a wire will have essentially normal polarization which makes plasmon phenomena noticeable for arbitrary light polarization.

Though the described spectral dependences of absorption are very specific and interesting, their experimental observation is more difficult than for the effects in semiconductor nanowires described in Sec. II A. In metals we can measure neither photoconductivity nor luminescence excitation and have to restrict ourselves to direct measurements of optical

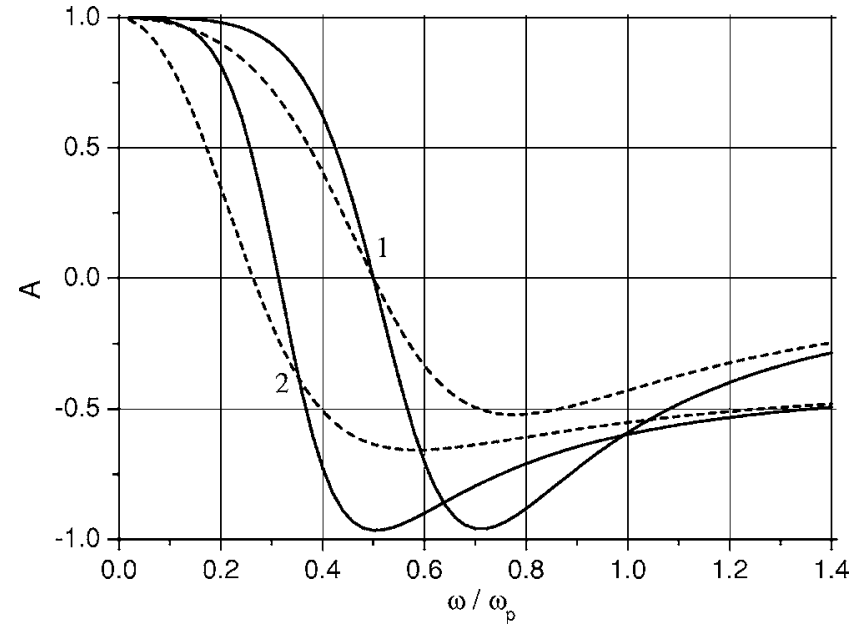

FIG. 2. Absorption anisotropy $A=\left(k_{\|}-k_{\perp}\right) /\left(k_{\|}+k_{\perp}\right)$ in metallic nanowires for $\varepsilon_{0}=\varepsilon_{\infty}$ (curves 1) and $\varepsilon_{0}=3 \varepsilon_{\infty}$ (curves 2). Solid curves correspond to $\nu=0.1 \omega_{p}$, dashed curves to $\nu=0.5 \omega_{p}$.

absorption. The most straightforward method to do it is to investigate large number of nanowires suspended in some liquid optically inactive in the spectral region of interest. In this case, orientation of nanowires is random, and no polarization dependence should be observed. Strong anisotropy of absorbing objects will be revealed only in a specific character of spectral dependence of the absorption coefficeint (Fig. $3)$. In this spectrum strong low-frequency absorption is caused by nanowires almost parallel to the light polarization while the plasmon maximum is formed by those with orientation close to the perpendicular one.

\section{Polarization of luminescence}

In the previous sections we considered physical phenomena related to the fact that external nonpolarized light becomes strongly polarized inside a nanowire with high refractive index. Now we discuss the related problem of

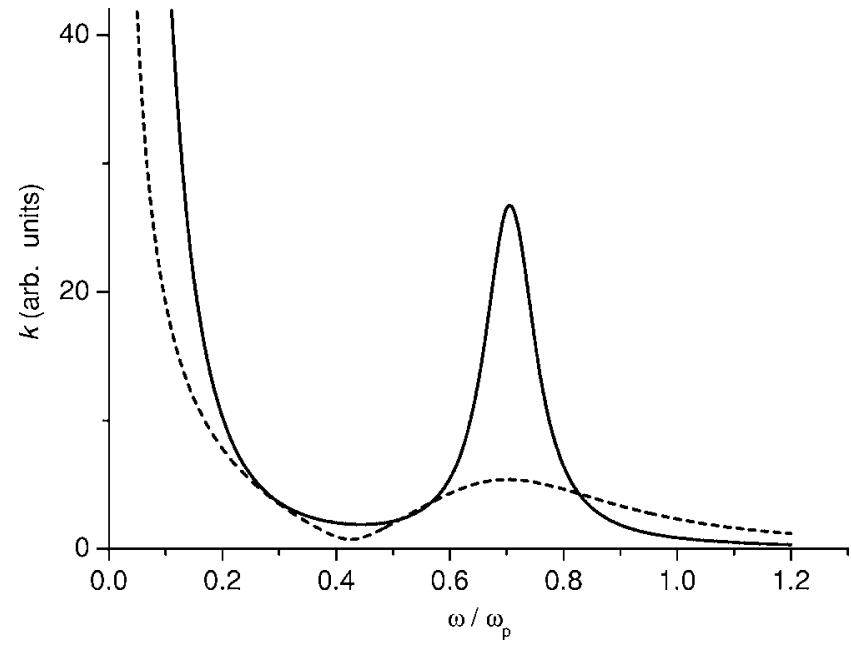

FIG. 3. Absorption spectrum of randomly oriented metallic nanowires. Solid curve corresponds to $\nu=0.1 \omega_{p}$, dashed curve to $\nu=0.5 \omega_{p}$. 
polarization of light emitted by a nanowire. As a first step, we solve the auxiliary problem of finding the electric field created by an electric dipole placed at the axis of a cylinder with radius $a$ and dielectric constant $\varepsilon$, in environment with $\varepsilon_{0}=1$. Details of these calculations are given in the Appendix for the more general case of a core-shell structure, and here we present only the final results, which for our particular case can be obtained from Eq. (A4) by assuming $r_{1}=r_{2}=a$. The result depends on the orientation of the dipole moment $\mathbf{d}_{0}$ relative to the cylinder axis ( $z$-axis), and we consider separately the cases $\mathbf{d}_{0} \| \mathbf{z}$ and $\mathbf{d}_{0} \perp \mathbf{z}$. In the first case, the electric field of the radiation far from the nanowire, has the same amplitude and configuration as would be created by a dipole with the moment $\mathbf{d}_{0}$ in free space [Eq. (A6)]. For the normal orientation of $\mathbf{d}$, the corresponding effective moment is given by $\mathbf{d}=2 \varepsilon_{0} \mathbf{d}_{0} /\left(\varepsilon+\varepsilon_{0}\right)$ [Eq. (A4)].

As a result, even for nanowires with isotropic interband matrix elements (which is definitely the case for cubic semiconductors and for moderate $a$ when size quantization only plays a minor role), where otherwise luminescence should be nonpolarized, it acquires a strong polarization under the influence of image forces.

As a second, we consider the net polarization of luminescence from a line $x, y=0$ containing a mixture of different emitting dipoles. We take some point $z$ along the wire axis containing three mutually perpendicular dipoles $d_{x}, d_{y}, d_{z}$ with frequency $\omega$ and calculate the resulting electric field far from the wire on the $x$-axis at some point $x_{0}$. We use the standard formula for the electric field of an emitting dipole in a wave zone and add contributions of all line segments by integrating over $z$. Since radiation from different segments is incoherent (see also the discussion in Sec. IV), we do not add electric fields, rather but Poynting vectors $\mathbf{S}$ containing no phase factors. The resulting $\mathbf{S}$ is directed along the $x$-axis and for the two different polarizations considered is

$$
\begin{gathered}
\left(S_{x}\right)_{\|}=\frac{\nu \omega^{4}}{6 \pi \varepsilon_{0}^{2} c^{3} x_{0}}\left(d_{x}^{2}+2 d_{z}^{2}\right), \\
\left(S_{x}\right)_{\perp}=\frac{\nu \omega^{4}}{2 \pi \varepsilon_{0}^{2} c^{3} x_{0}} d_{y}^{2},
\end{gathered}
$$

where $\nu$ is the density of dipoles per unit line length.

Combining these two problems and substituting $d_{x, y}$ $=2 \varepsilon_{0} d_{0 x . y} /\left(\varepsilon+\varepsilon_{0}\right) ; d_{z}=d_{0 z}$ into Eq. (7), provides the resulting polarization of light emitted by a nanowire. The intensity ratio for different light polarization in the case of isotropic matrix elements is

$$
\frac{I_{\|}}{I_{\perp}}=\frac{\left(\varepsilon+\varepsilon_{0}\right)^{2}+2 \varepsilon_{0}^{2}}{6 \varepsilon_{0}^{2}} .
$$

If the bare dipole moment components $d_{0 x . y, z}$ do not coincide due to size quantization or/and crystal anisotropy, ${ }^{6,7}$ this modifies polarization character introducing anisotropy even in the absence of image forces, similar to the case of absorption (Sec. II A).

It is seen from Eq. (8) that light emitted by a nanowire with a dielectric constant higher than that of its environment, will be strongly polarized in the wire direction, which was clearly demonstrated experimentally ${ }^{11,12}$ (in a number of other works ${ }^{8-10,15}$ such polarization was also observed but was presumably caused by the above-mentioned anisotropy of matrix elements, rather than by image forces). The effect is qualitatively similar to the property of absorption considered in Sec. II A but the magnitude of the polarization is different. By comparing Eq. (3) with Eq. (8) one can see that the degree of polarization for luminescence is less than for absorption. It is caused by the fact that, according to Eq. (7), light with parallel polarization is generated not only by $d_{z}$ dipole moments but also by $d_{x}$ which are weakened by image forces. For example, for InP nanowires $(\varepsilon=12.7)$ where, as was already mentioned in Sec. II A, $k_{\|} / k_{\perp} \simeq 49$, Eq. (8) gives $I_{\|} / I_{\perp} \simeq 30$, in good agreement with the experimentally observed values of $k_{\|} / k_{\perp} \simeq 48$ and $I_{\|} / I_{\perp} \simeq 24{ }^{11}$

\section{Polarization memory}

So far we have considered luminescence from a single nanowire. In many cases real nanowire structures are comprised of arrays of many parallel nanowires and the luminescence is a sum of their individual contributions. If all the nanowires are parallel, Eq. (8) can adequately describe the behavior of such an whole array since the terms "parallel" or "perpendicular" polarization have the same meaning for all nanowires. We implicitly used this fact in the previous paragraph when comparing the single-wire formula Eq. (8) with experimental data for systems of parallel nanowires.

In the case of an array of randomly oriented nanowires (say, nanowires in a polymer matrix of in solution), the system has macroscopically isotropic optical properties but simultaneously must possess a very interesting property of polarization memory. If we excite photoluminescence in the system using polarized light, in accordance with Sec. II A, nonequlibrium carriers will be generated mostly in the nanowires that are oriented close to parallel to the light polarization. According to Sec. II C, light emitted by these nanowires will have preferable polarization parallel to them, or in other words, parallel to the polarization of the exciting radiation (a similar idea was suggested in Ref. 16 for explanation of the polarized luminescence in porous Si observed in Ref. 17). If the nanowires are not fixed in space but can vary their orientation (as in solution) then to observe the polarization memory we require that the characteristic time of wire reorientation is much larger than the lifetime of the optically generated carriers.

To calculate this effect, we assume that the nanowire array occupies the semispace $x<0$ and study the polarization of the net luminescence excited by $z$-polarized light and measured at some point $\left(x_{0}, 0,0\right)$ outside the array (that is, $x_{0}>0$ ). By symmetry, the resulting Poynting vector $\mathbf{S}$ at this point is directed along $x$-axis so that we must add contributions to $\left(S_{x}\right)_{z}$ and $\left(S_{x}\right)_{y}$ (the last subscript indicates direction of polarization) from wires with all possible orientations and compare the resulting sums. Let us consider some nanowire with orientation characterized by the spherical angles $(\theta, \alpha)$ (see Fig. 4). The Poynting vector of its radiation $\mathbf{S}$ is perpendicular to the wire and the ratio of electric field components $\left(E_{\|} / E_{\perp}\right)^{2}=I_{\|} / I_{\perp}$ is given by Eq. (8). To perform summation 


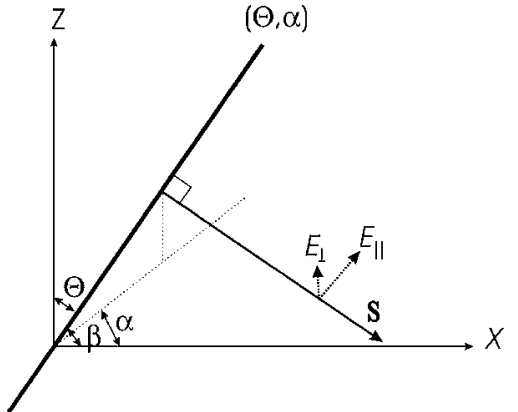

FIG. 4. Electric field and Poynting vectors created by a nanowire with arbitrary orientation.

over all nanowires, we must transform these characteristics to a Cartesian coordinate system. Taking into account that in Fig. 4, $\cos \beta=\sin \theta \cos \alpha$, we get

$$
S_{x}=S \sqrt{1-\sin ^{2} \theta \cos ^{2} \alpha},
$$

$E_{\| x}=E_{\|} \sin \theta \cos \alpha, \quad E_{\| y}=E_{\|} \sin \theta \sin \alpha, \quad E_{\| z}=E_{\|} \cos \theta$,

$$
\begin{gathered}
E_{\perp x}=0, \quad E_{\perp y}=-\frac{E_{\perp} \cos \theta}{\sqrt{\cos ^{2} \theta+\sin ^{2} \theta \sin ^{2} \alpha}}, \\
E_{\perp z}=\frac{E_{\perp} \sin \theta \sin \alpha}{\sqrt{\cos ^{2} \theta+\sin ^{2} \theta \sin ^{2} \alpha}} .
\end{gathered}
$$

Equations (10) and (11) give

$$
\begin{aligned}
\frac{\left(S_{x}\right)_{z}}{\left(S_{x}\right)_{y}} & =\frac{E_{\| z}^{2}+E_{\perp z}^{2}}{E_{\| y}^{2}+E_{\perp y}^{2}} \\
& =\frac{E_{\|}^{2} \cos ^{2} \theta\left(\cos ^{2} \theta+\sin ^{2} \theta \sin ^{2} \alpha\right)+E_{\perp}^{2} \sin ^{2} \theta \sin ^{2} \alpha}{E_{\|}^{2} \sin ^{2} \theta \sin ^{2} \alpha\left(\cos ^{2} \theta+\sin ^{2} \theta \sin ^{2} \alpha\right)+E_{\perp}^{2} \cos ^{2} \theta} .
\end{aligned}
$$

The averaging should be performed over all nanowire orientations by integration numerator and denominator of Eq. (12) over $\theta$ and $\alpha$. If $E_{\|}$and $E_{\perp}$ were the same for all nanowires, the answer would be equal to one (no polarization). However, according to Eq. (3), the intensity of absorbed exciting light and hence the intensity of luminescence depends on $\theta$ being proportional to $\left(\varepsilon+\varepsilon_{0}\right)^{2} \cos ^{2} \theta+4 \varepsilon_{0}^{2} \sin ^{2} \theta$. Including this weighting factor in the integration and using Eq. (8) for $\left(E_{\|} / E_{\perp}\right)^{2}$, we obtain

$$
\frac{I_{\|}}{I_{\perp}}=\frac{9\left[\left(\varepsilon+\varepsilon_{0}\right)^{2}+2 \varepsilon_{0}^{2}\right]\left(\varepsilon+\varepsilon_{0}\right)^{2}+20\left[\left(\varepsilon+\varepsilon_{0}\right)^{2}+2 \varepsilon_{0}^{2}\right]+21\left(\varepsilon+\varepsilon_{0}\right)^{2}+336 \varepsilon_{0}^{2}}{3\left[\left(\varepsilon+\varepsilon_{0}\right)^{2}+2 \varepsilon_{0}^{2}\right]\left(\varepsilon+\varepsilon_{0}\right)^{2}+44\left[\left(\varepsilon+\varepsilon_{0}\right)^{2}+2 \varepsilon_{0}^{2}\right]+63\left(\varepsilon+\varepsilon_{0}\right)^{2}+168 \varepsilon_{0}^{2}}
$$

(note that in this formula the subscripts $\|$ and $\perp$ indicate polarization of luminescence with respect to the polarization of the exciting light).

Equation (13) shows that maximal possible polarization, reached at $\left|\varepsilon / \varepsilon_{0}\right| \gg 1$, is equal to $I_{\|} / I_{\perp}=3$. In terms of the polarization ratio $P=\left(I_{\|}-I_{\perp}\right) /\left(I_{\|}+I_{\perp}\right)$ it corresponds to $P=0.5$. Figure 5 shows this polarization ratio for an arbitrary relationship between $\varepsilon$ and $\varepsilon_{0}$. For InP nanowires in air $(\varepsilon=12.7)$ we get $P=0.44$. It is worth noting that at $\varepsilon<\varepsilon_{0}$ (e.g., in metallic nanowires near the plasmon resonance), when electric field in nanowires has a preferably perpendicular orientation, the system also has a polarization memory. This effect is weaker than at large $\varepsilon$, with the maximum $P=6 / 68=0.088$.

\section{CORE-SHELL NANOWIRES}

\section{A. Potential distribution}

Now we generalize some of the results of Sec. II A to the case of core-shell nanowires containing two coaxial regions with different dielectric constants. Fabrication of such structures has been demenstrated experimentally. ${ }^{18,19}$

We consider a core-shell wire consisting of a central core having radius $r_{1}$ and dielectric constant $\varepsilon_{1}$ and the shell with radius $r_{2}>r_{1}$ and dielectric constant $\varepsilon_{2}$. Calculations similar to those performed in Ref. 20, show that an external electric field $\mathbf{E}_{0}$ perpendicular to the wire axis creates in the core a uniform field

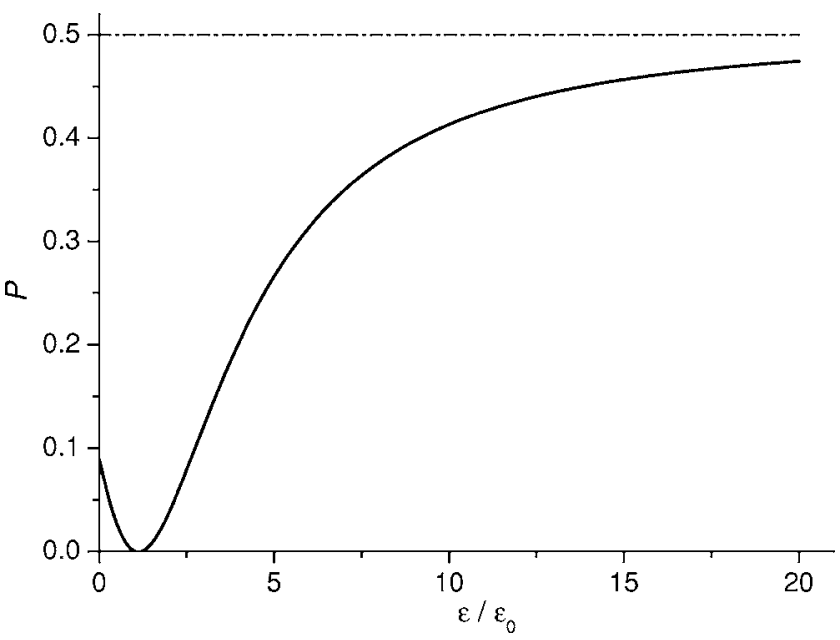

FIG. 5. Luminescence polarization ratio in a random system of nanowires exited by polarized light. The dotted-and-dashed line corresponds to the limiting value $P=0.5$. 


$$
\mathbf{E}_{1}=\frac{4 \varepsilon_{0} \varepsilon_{2} \mathbf{E}_{0}}{2 \varepsilon_{2}\left(\varepsilon_{1}+\varepsilon_{0}\right)+p\left(\varepsilon_{2}-\varepsilon_{1}\right)\left(\varepsilon_{2}-\varepsilon_{0}\right)} \equiv A \mathbf{E}_{0}
$$

where $p=1-\left(r_{1} / r_{2}\right)^{2}$ is the ratio of shell volume to total nanowire volume and $A$ is the same amplification factor as that for a dipole field in the structure calculated in the Appendix. The field in the shell is a sum of a uniform field and that of a linear dipole,

$$
\begin{aligned}
\mathbf{E}_{2}= & \frac{2 \varepsilon_{0}}{2 \varepsilon_{2}\left(\varepsilon_{1}+\varepsilon_{0}\right)+p\left(\varepsilon_{2}-\varepsilon_{1}\right)\left(\varepsilon_{2}-\varepsilon_{0}\right)}\left\{\left(\varepsilon_{1}+\varepsilon_{2}\right) \mathbf{E}_{0}\right. \\
& \left.+2\left(\varepsilon_{1}-\varepsilon_{2}\right)(1-p) r_{1}^{2}\left[\frac{\mathbf{E}_{0}}{r^{2}}-\frac{2 \mathbf{r}\left(\mathbf{r} \mathbf{E}_{0}\right)}{r^{4}}\right]\right\} .
\end{aligned}
$$

It is worth noting that solution of the Laplace equation resulting in Eq. (15) assumed local connection between the values of electric field $\mathbf{E}_{2}$ and electrical induction $\varepsilon_{2} \mathbf{E}_{2}$ which will be the case if the mean free path of electrons in a metallic shell is less than its thickness $r_{2}-r_{1}$.

For $\mathbf{E}_{0}$ parallel to the wire axis, $\mathbf{E}_{1}=\mathbf{E}_{2}=\mathbf{E}_{0}$.

Equations (14) and (15) can be used to determine the spectra and polarization dependence of optical absorption in core-shell nanowires. We will not analyze the cases when the core and the shell are both semiconducting or both metallic since they have no qualitatative difference with the effects considered, respectively, in Secs. II A and II B. The situation is different for nanowires with a metallic shell and a semiconductor (or dielectric) core. As we show below, the plasmon spectra of such systems differ noticeably from those in uniform metallic nanowires and, besides, mutual influence of plasmon effects in a shell and luminescent phenomena in a semiconductor core results in new effects and new functional possibilities of core-shell structures.

The specific features of the optical response of metallic shells arises from the fact that the plasmon-related absorption maximum existing in the entire metallic shell is split into two maxima with positions depending on the relationship between $r_{1}$ and $r_{2}$ for the light with perpendicular polarization (Sec. II B) (the effect is well known for core-shell nanodots, see, e.g., Ref. 14). The frequency regions near the plasmon resonances are characterized by anomalously high electric field strength (light amplification) both in the core and in the shell. As it has been already pointed out for coreshell nanodots, ${ }^{20}$ this can be used for enhancement of nonlinear optical effects in a core (in our case, only for light with a perpendicular polarization). There is also a reciprocal effect: an oscillating dipole $\mathbf{d}_{0}$ in the core creates far from the wire radiation field which may be considerably larger than that in a uniform medium. This will be considered later in Sec. III C. In the next section, we investigate optical absorption near the plasmon resonances caused by the increased electric field $\mathbf{E}_{2}$ in the shell.

\section{B. Spectra and polarization dependence of absorption}

To calculate the optical absorption in a core-shell nanowire caused by light with perpendicular polarization, we must include the local absorption $\operatorname{Im} \varepsilon_{2}\left|\mathbf{E}_{2}(r)\right|^{2}$ and integrate it over the whole shell. Using the Drude formula for $\varepsilon_{2}$ and Eq. (15) for $\mathbf{E}_{2}(r)$, we obtain the absorption spectra shown by solid lines in Fig. 6. These are seen to contain two maxima corresponding to plasmon resonances where the real part of the denominator in Eqs. (14) and (15) vanishes and the electric field in the wire becomes anomalously large. With increasing $p$, the maxima approach each other and the low-frequency one diminishes so that at $p \rightarrow 1$ we get a single peak at $\omega=\omega_{p} / \sqrt{2}$ corresponding to the plasma resonance in a long cylinder.

Similar phenomena in core-shell nanodots have been already observed experimentally ${ }^{21,22}$ and have a good theoretical description. ${ }^{20,23}$ It is important to emphasize that, contrary to nanodots, the described two-peak absorption spectra in nanowires are observed only for light polarized perpendicularly to nanowires. For parallel polarization, when electric field in nanowires is equal to the external one, we have a simple monotonically decreasing spectra reflecting the lowfrequency absorption given by $\operatorname{Im} \varepsilon_{2}$.

If the core is a semiconductor with the band gap $E_{g}<\hbar \omega$ then, besides the absorption described above, there is also interband absorption in the core proportional to $\left|\mathbf{E}_{1}\right|^{2}$. The spectrum of this absorption can be easily calculated from Eq. (14) and is shown by dashed lines in Fig. 6. The spectra also have a double maximum structure qualitatively similar to that for a metallic shell but, contrary to that, suffering a dramatic drop with increasing $\varepsilon_{1}$ due to the extrusion of electric field from the high- $\varepsilon$ core.

We must emphasize that magnitudes of absorption in a shell and a core depend on different parameters (i.e., the plasma frequency and scattering rate in metals, and band gap width and interband matrix element in semiconductors) and thus we cannot compare the amplitudes of solid and dashed curves and say a priori what type of absorption is dominating. Moreover, it is also difficult to distinguish experimentally these two absorption components since the absorption spectra in a shell and a core have qualitatively similar characters. The problem can be solved by measuring the excitation spectrum for core luminescence. Those are proportional only to the partial absorption in the core shown by the dashed lines in the figure.

As was mentioned above, the dashed lines in Fig. 6 actually show the spectral dependence of the electric field in a core $\left|\mathbf{E}_{1}\right|$. As we have shown in the previous sections, semiconductor and metallic nanowires have an opposite character of electrical anisotropy: the perpendicular component of the electric field is suppressed as compared with the parallel one, in a semiconductor nanowire, and enhanced in a metallic nanowire in the vicinity of a plasmon frequency. In composite core-shell structures, both variants can be realized, depending on the shell thickness and light frequency. The corresponding "phase diagram" using $p-\omega$ axes, is shown in Fig. 7. The areas above the line(s) correspond to the "metallic" case $E_{\perp}>E_{0 \perp}$ while those below the line(s) - to the "semiconductor" case $E_{\perp}<E_{0 \perp}$. In other words, these lines correspond to $|A|=1$ where the amplification factor $A \equiv E_{\perp} / E_{0 \perp}$ is given by Eq. (14). At small $p$, one naturally deals with the 

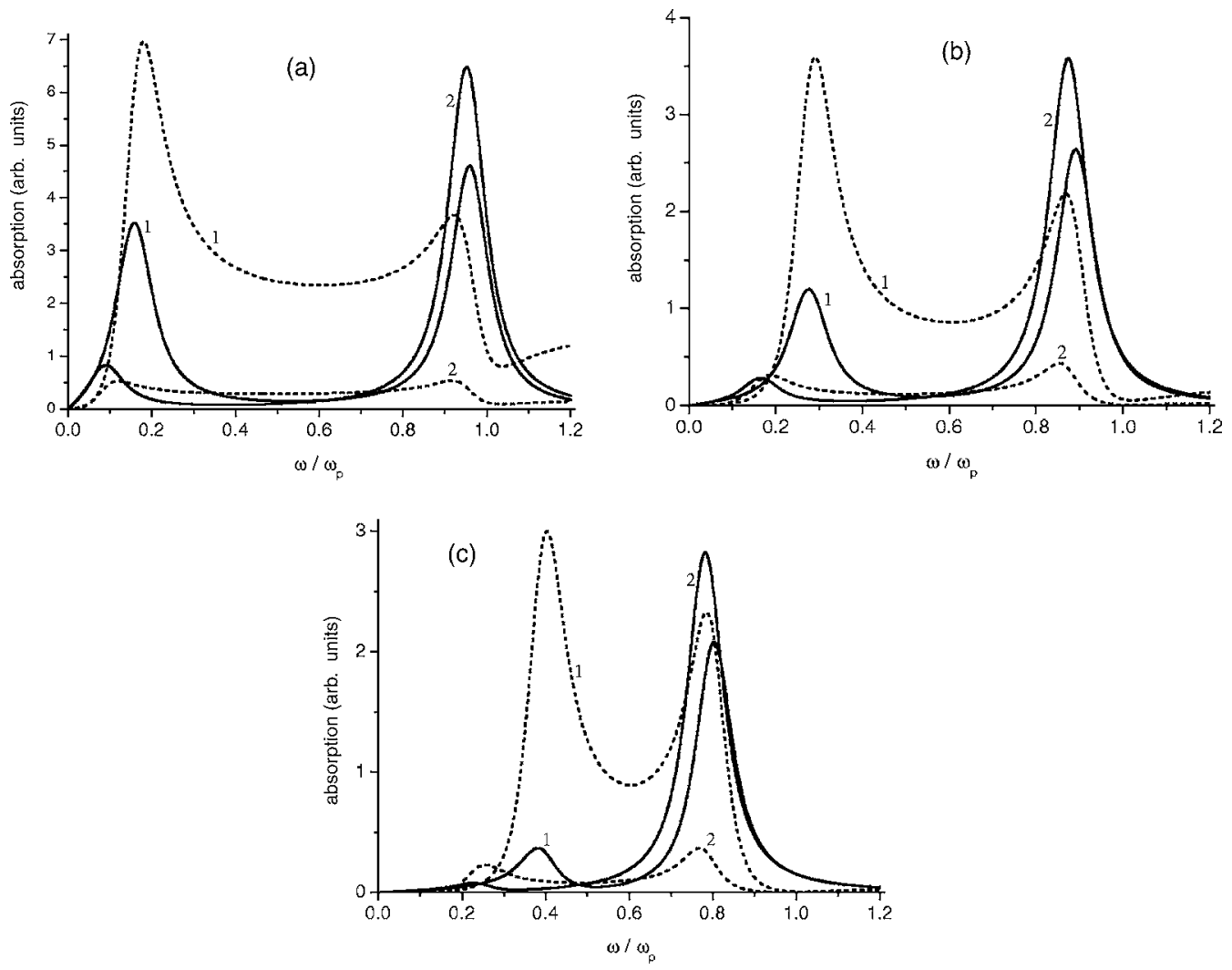

FIG. 6. Absorption spectra of nanowires with a metallic shell for $p=0.2$ (a), 0.5 (b), 0.8 (c) and $\nu / \omega_{p}=0.1$ and a semiconductor core with $\varepsilon_{1}=3$ (curves 1) and $\varepsilon_{1}=10$ (curves 2) for light polarized perpendicularly to nanowires. Solid curves correspond to absorption in a metallic shell, dashed curves in a semiconductor core.

"semiconductor" regime. One might be surprised that at $p \rightarrow 1$ the regime is not "metallic" but it should be pointed that the figure describes the field, not in a metallic shell, but in a semiconductor core, which in the limit of a pure metallic nanowire $p \rightarrow 1$ simply disappears.

The condition $|A|>1$ is satisfied in the vicinity of plasmon resonances which means that for light inducing interband absorption in the core, the intensity of electron-hole

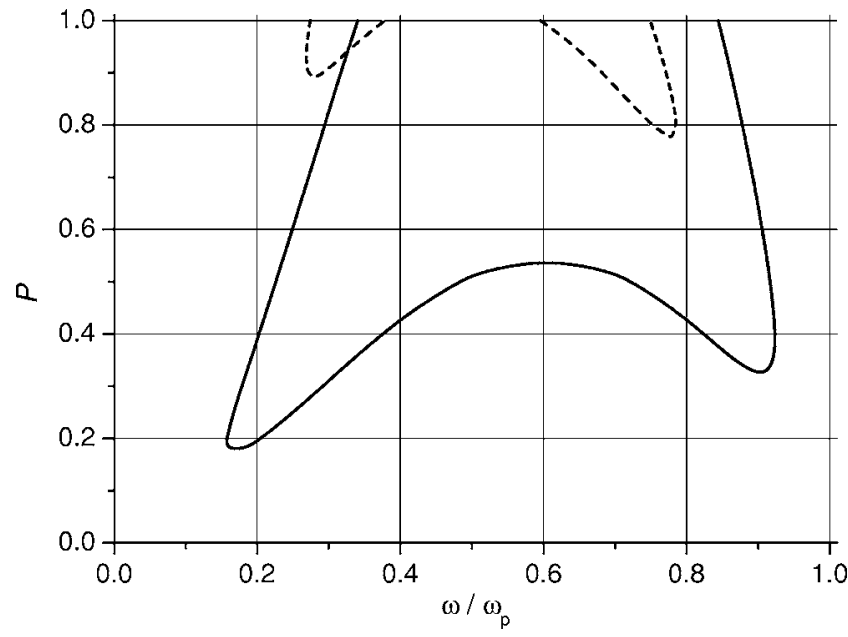

FIG. 7. Values of $p$ and $\omega$, for which $E_{\perp}=E_{0 \perp}$ at $\varepsilon_{1}=3$ (solid line) and $\varepsilon_{1}=10$ (dashed lines). The area above the line(s) corresponds to $E_{\perp}>E_{0 \perp}$. pair generation and, hence, the intensity of luminescence caused by these pairs is larger than in the absence of a metallic shell. That is why we use for $A$ the term "amplification factor."

\section{Luminescence amplification}

Metallic shells may cause amplification not only of the ac electric field of the exciting light due but also of the light emitted by a semiconductor core. The ac electric field emitted by a core, which can be considered as an effective dipole $\mathbf{d}_{0}$, is disturbed by a metallic shell so that the field far from the nanostructure looks like one created by some other effective dipole $\mathbf{d}$. By analogy with the previous section, we may expect that in the vicinity of a plasmon resonance the condition $\left|d / d_{0}\right| \gg 1$ can be realized, which means an effective amplification of the emitted radiation as well.

The calculations presented in the Appendix show that luminescence of core-shell structures is characterized by exactly the same rules as absorption. The field of dipoles parallel to the nanowire axis is not disturbed by image forces, while the field of perpendicular dipoles acquires the additional factor $A$. As a result, for an isotropic distribution of effective dipoles, such as in a cubic semiconductor core in the absence of size quantization, the emission from a nanowire acquires a partial polarization parallel to the wire axis at $|A|<1$ (as in Sec. II C) or perpendicular at $|A|>1$. Since $A$ 

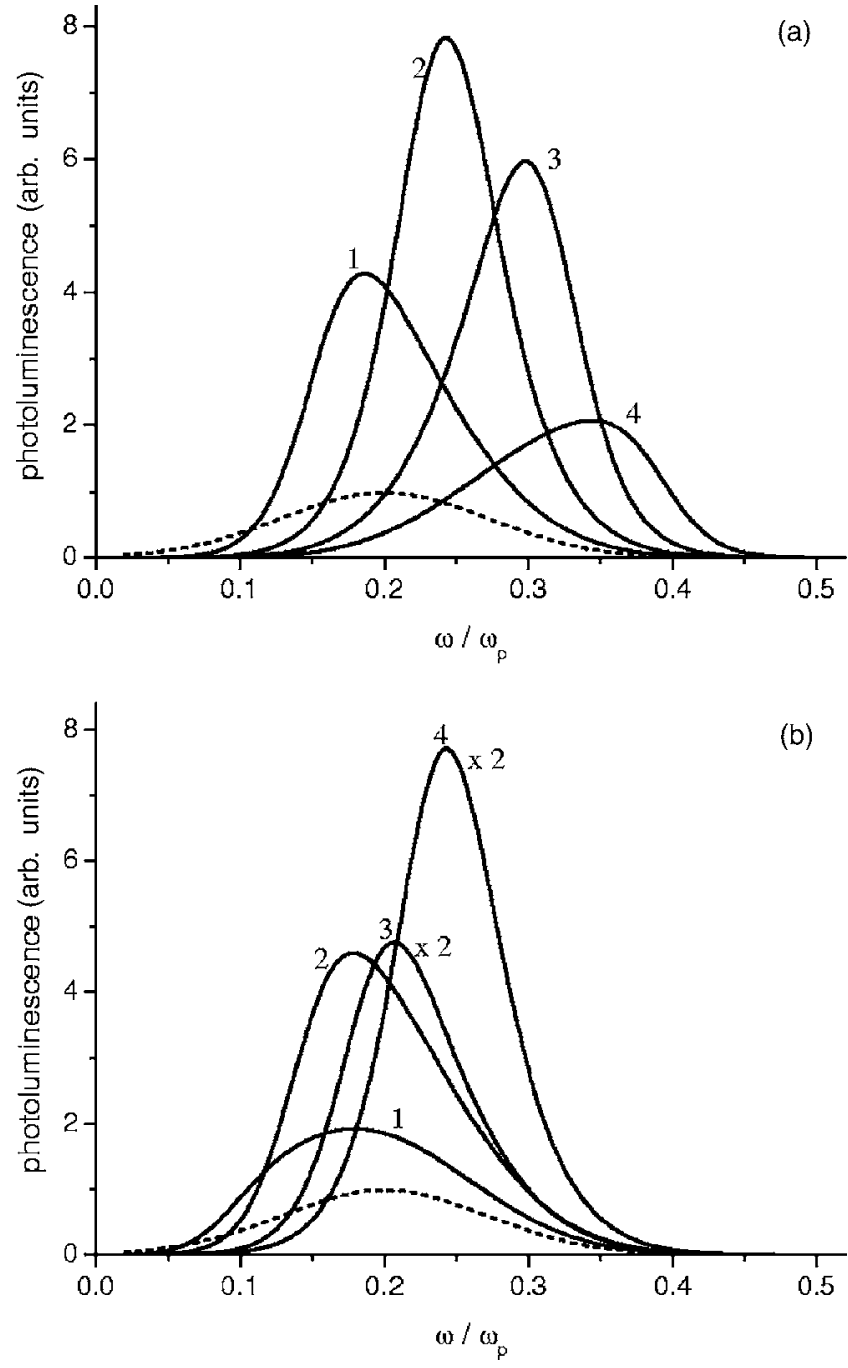

FIG. 8. Amplification and spectral shift of a luminescence line with $\omega_{\mathrm{em}}=0.2 \omega_{p}$ for an effective dipole perpendicular to the axis of a core-shell nanowire with $\varepsilon_{1}=3$ (a) and $\varepsilon_{1}=10$ (b). Dashed lines correspond to the absence of a metallic shell $(p=0)$, solid lines to a different relative volume of a shell: $1-p=0.2 ; 2-p=0.4 ; 3-p=0.6$; $4-p=0.8$.

has a strong frequency dependence, the components of emitted light with different polarization may have different spectra depending on the metallic shell parameters.

For an effective dipole $d_{0 x}$ perpendicular to the axis of a core-shell nanowire, the real luminescence spectrum is determined by the product of the bare luminescence spectrum $\left|d_{0 x}(\omega)\right|^{2}$ and the spectrum of $|A(\omega)|^{2}$. To demonstrate the role of a metallic shell, we performed calculations assuming $\left|d_{0 x}(\omega)\right|^{2}$ to have a Gaussian shape with the center at $\omega_{\mathrm{em}}$ $=0.2 \omega_{p}$ and the width $0.1 \omega_{p}$. One can see from Fig. 6 that for reasonable parameters of nanoshells, the lower plasmon peak may lie both left and right of this $\omega_{\mathrm{em}}$. Figure 8 shows the resulting transformation of the luminescence spectrum for perpendicular to a nanowire polarization. The most remarkable but anticipated result consists of a dramatic increase in the luminescence intensity near the resonance condition when the plasmon frequency coincides with $\omega_{\mathrm{em}}$. Another interesting feature is the shifting of the frequency of the luminescence line in off-resonant conditions. Convolution of a bare luminescence line with the spectrum of the amplification factor may result in a nonmonotonic behavior of the resulting spectrum shown in Fig. 8(b), when an increase in the nanoshell thickness (which grows with the curve number) may cause first a red and then a blue shift of the luminescence line. From the practical point of view, the results of Fig. 8 demonstrate that by using metallic shells we can increase multifold the intensity of nanowire luminescence and shift the spectral position of a luminescent line. Qualitatively similar effects for core-shell nanowires have been recently predicted in Ref. 23.

If we have an array of randomly oriented wires, the resulting luminescence spectrum will be, of course, nonpolarized and determined by a mixture of contributions from effective dipole with parallel and perpendicular orientations with the spectra given, respectively, by the dashed and the corresponding solid line in Fig. 8. Since in the most interesting case when $\omega_{\mathrm{em}}$ is close to the plasmon resonance, the latter has much larger amplitude than the former (see the figure), the emission spectrum of an array will be given practically by the same solid line.

It would be very attractive to enhance the luminescence dramatically by a simultaneous amplification of both exciting and emitting light. Such a situation often occurs in surfaceenhanced Raman scattering ${ }^{24}$ but in luminescence its realization is more difficult since the difference in frequencies of exciting $\omega_{\mathrm{ex}}$ and emitted $\omega_{\mathrm{em}}$ light is usually quite large. They can be covered by a single plasmon peak only if the latter is wide enough, which can be realized only at large enough $\nu$ which corresponds to a low level of amplification. An alternative possibility consists in using both plasmon peaks shown in Fig. 6. In this case the shell parameters should be chosen in such a way that the low-frequency peak corresponds to $\omega_{\mathrm{em}}$ in a given nanowire core while the source of excitation is tuned for $\omega_{\mathrm{ex}}$ to coincide with the second peak lying near $\omega_{p}$.

The situation may exist when the frequency of the exciting light corresponds to $|A|>1$ and that of emitted light corresponds to $|A|<1$, or vice versa. It has a lesser practical importance for light emitters but is characterized by an interesting property to some extent opposite to that considered in Sec. II D and could be called polarization "antimemory." Consider an array of randomly oriented nanowires with the above-mentioned properties. At the frequency of excitation $\left|A\left(\omega_{\mathrm{ex}}\right)\right|>1$, nonequilibrium carriers are generated mostly in the nanowires almost perpendicular to the light polarization. If, at the same time, at the frequency of luminescence $\left|A\left(\omega_{\mathrm{em}}\right)\right|<1$, then the emitted light will have a preferable polarization parallel to their axes, that is perpendicular to the polarization of excitation.

To consider this effect analytically, we must modify calculations for the polarization memory presented in Sec. II D. Namely, by the ratio $E_{\|} / E_{\perp}$ in the expression Eq. (12) we mean $1 / A\left(\omega_{\mathrm{em}}\right)$ calculated at the frequency of emission while the angular integration must be performed using the 

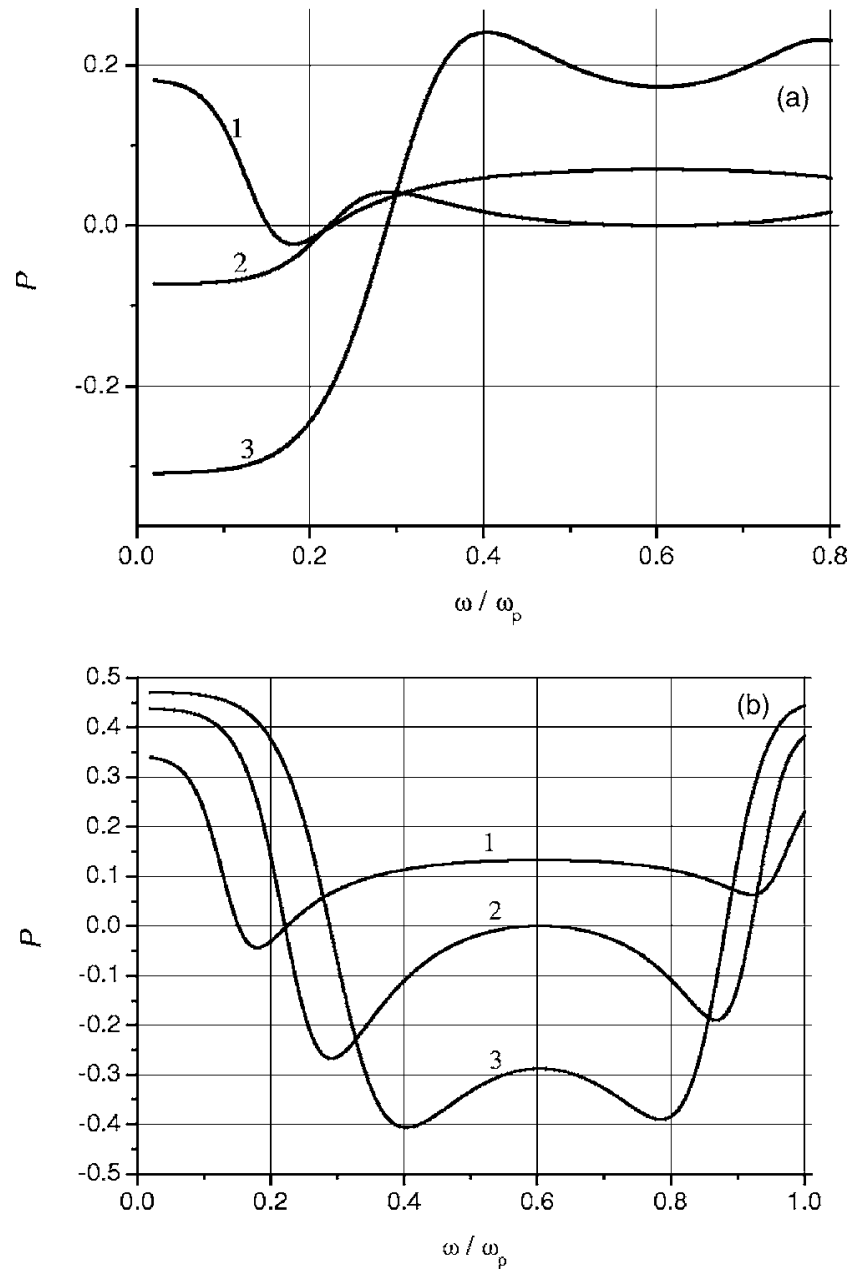

FIG. 9. Luminescence polarization ratio in a random system of core-shell nanowires with $\varepsilon_{1}=3$ excited by a polarized light with $\omega=0.8 \omega_{p}$ (a) and $\omega=\omega_{p}$ (b). $1-p=0.2 ; 2-p=0.5 ; 3-p=0.8$.

weighting factor $\cos ^{2} \theta+\left|A\left(\omega_{\text {ex }}\right)\right|^{2} \sin ^{2} \theta$ taken at the frequency of excitation. This gives us

$$
\frac{I_{\|}}{I_{\perp}}=\frac{18+10\left|A\left(\omega_{\mathrm{ex}}\right)\right|^{2}+7\left|A\left(\omega_{\mathrm{em}}\right)\right|^{2}+28\left|A\left(\omega_{\mathrm{ex}}\right) A\left(\omega_{\mathrm{em}}\right)\right|^{2}}{6+22\left|A\left(\omega_{\mathrm{ex}}\right)\right|^{2}+21\left|A\left(\omega_{\mathrm{em}}\right)\right|^{2}+14\left|A\left(\omega_{\mathrm{ex}}\right) A\left(\omega_{\mathrm{em}}\right)\right|^{2}} .
$$

Figure 9 drawn on the base of Eq. (16) shows the spectral dependence of the luminescence polarization in a random array of nanowires for different choice of parameters. Calculations were performed for two different excitation frequencies: $\omega=\omega_{p}$, where $\left|A\left(\omega_{\mathrm{ex}}\right)\right|^{2}$ is always $<1$, and $\omega=0.8 \omega_{p}$ where, according to Fig. $8,\left|A\left(\omega_{\text {ex }}\right)\right|^{2}>1$ at large $p$. It is seen that the same structure may demonstrate both "memory" $(P>0)$ and "antimemory" $(P<0)$ in the different spectral regions with boundaries determined by Fig. 7 .

\section{DISCUSSION}

We analyzed the effects of optical anisotropy in cylindrical nanowires surrounded by air or embedded into a medium with essentially different dielectric constant. Similar effects can be also observed in more complicated nanostructures, e.g., in nanowires obtained by submicron lithography of quantum well heterostructures. In this case optical anisotropy should be present as well but has a more complicated character since the quasi-one-dimensional electron system is surrounded by air only from two sided while from two other sides it borders upon wide-gap semiconductor with almost the same $\varepsilon$. In this case, the calculation of electric field and optical anisotropy can be done only numerically, which was partially performed in Ref. 25.

We want to emphasize that the whole theory presented above was of a purely classical character with: (i) ignoring size quantization phenomena and (ii) assuming that different wire segment emit independently and incoherently. Such an approach is adequate if either the quantization energy $\hbar^{2} /\left(m a^{2}\right)$ ( $m$ is the effective mass of carriers) is less than $k T$ or if the mean free path of carriers is less than the nanowire radius $a$. In typical experiments (e.g., Refs. 11,12) where $a$ varies from several tens to hundred nanometers, this condition should be filfilled. In thinner nanowires we may expect our predictions to be still valid qualitatively (though not quantitative) with some additional anisotropy of emission and absorption caused by size quantization.

In Sec. II B we discussed optical properties of nanowires with a uniform continuous metallic shell. Recently, first communications have also appeared describing fabrication of semiconductor nanowires covered by disconnected metallic nanoparticles. They can be described by Eqs. (14) and (15) as well, if, instead of $\varepsilon_{2}$, we use an effective dielectric constant of a statistical metal-air mixture calculate, e.g., in the framework of the effective medium theory. ${ }^{26}$ Earlier, we used such an approach for calculating optical properties of nanodots with a discontinuous metallic shells. ${ }^{23}$

In conclusion we must note that all our calculations were based on simple formulas for electric field in a single cylindrical inclusion and for this reason may become inadequate for high density nanowire arrays when the distance between wires becomes comparable with their diameter.

\section{ACKNOWLEDGMENTS}

The authors gratefully acknowledge financial support from NSERC, CIPI, MMO, CITO, CSA, and AFOSR in carrying out this research.

\section{APPENDIX}

In cylindrical coordinates $(\rho, z, \alpha)$ the potential of a dipole with the moment $d_{0}=d_{0 x}$ (perpendicular to the cylinder axis) in a uniform medium with the dielectric constant $\varepsilon_{0}$ is $\varphi_{0}^{(x)}$ $\times(\rho, z, \alpha)=d_{0 x} \rho \cos \alpha /\left[\varepsilon_{0}\left(\rho^{2}+z^{2}\right)^{3 / 2}\right]$. Due to the cylindrical symmetry, we may expect the total potential with the account of image forces to be also proportional to $\cos \alpha$. Using the well-known general expressions for nonsingular solutions of the Poisson equation in cylindrical coordinates and taking into account that potential must be even in $z$, we can write the total potential for a core-shell structure determined in Sec. III, in the form 


$$
\varphi^{(x)}(\rho, z, \alpha)=\left\{\begin{array}{cl}
\frac{d_{0 x} \rho \cos \alpha}{\varepsilon_{1}\left(\rho^{2}+z^{2}\right)^{3 / 2}}+\cos \alpha \int_{0}^{\infty} A(\lambda) I_{1}(\lambda \rho) \cos (\lambda z) d \lambda, & \rho<r_{1} ; \\
\cos \alpha \int_{0}^{\infty}\left[B(\lambda) I_{1}(\lambda \rho)+C(\lambda) K_{1}(\lambda \rho)\right] \cos (\lambda z) d \lambda, & r_{1}<\rho<r_{2} ; \\
\cos \alpha \int_{0}^{\infty} D(\lambda) K_{1}(\lambda \rho) \cos (\lambda z) d \lambda, & \rho>r_{2},
\end{array}\right.
$$

where $I_{1}$ and $K_{1}$ are the Bessel functions of an imaginary argument. The functions $A, B, C, D$ are to be found from matching the potential and its normal derivative at the interfaces $\rho=r_{1}, r_{2}$. Taking into account that

$$
\frac{\rho}{\left(\rho^{2}+z^{2}\right)^{3 / 2}}=\frac{2}{\pi} \int_{0}^{\infty} \lambda K_{1}(\lambda \rho) \cos (\lambda z) d \lambda,
$$

we can write these conditions in the form

$$
\begin{gathered}
\frac{2 \lambda d_{0 x}}{\pi \varepsilon_{1}} K_{1}\left(\lambda r_{1}\right)+A(\lambda) I_{1}\left(\lambda r_{1}\right)=B(\lambda) I_{1}\left(\lambda r_{1}\right)+C(\lambda) K_{1}\left(\lambda r_{1}\right) \\
\frac{2 \lambda d_{0 x}}{\pi} K_{1}^{\prime}\left(\lambda r_{1}\right)+\varepsilon_{1} A(\lambda) I_{1}^{\prime}\left(\lambda r_{1}\right)=\varepsilon_{2} B(\lambda) I_{1}^{\prime}\left(\lambda r_{1}\right) \\
+\varepsilon_{2} C(\lambda) K_{1}^{\prime}\left(\lambda r_{1}\right) ; \\
B(\lambda) I_{1}\left(\lambda r_{2}\right)+C(\lambda) K_{1}\left(\lambda r_{2}\right)=D(\lambda) K_{1}\left(\lambda r_{2}\right) ; \\
\varepsilon_{2} B(\lambda) I_{1}^{\prime}\left(\lambda r_{2}\right)+\varepsilon_{2} C(\lambda) K_{1}^{\prime}\left(\lambda r_{1}\right)=\varepsilon_{0} D(\lambda) K_{1}^{\prime}\left(\lambda r_{2}\right) .
\end{gathered}
$$

The object of our primary interest is $D(\lambda)$ determining the light emitted outside. Since Eq. (A2) represents a standard linear system, the exact solution for $D(\lambda)$ can be readily obtained but is very cumbersome and for this reason not presented here. What we need in fact, is the behavior of $\varphi^{(z)}(\rho, z)$ at large $\rho$ and $z$ (in the wave zone), which is determined by $D(\lambda)$ at small arguments, $\lambda r_{2} \ll 1$. After using the small-argument asymptotic for the Bessel functions: $I_{1}(x)$ $\simeq x / 2 ; I_{1}^{\prime}(x) \simeq 1 / 2 ; K_{1}(x) \simeq 1 / x ; K_{1}^{\prime}(x) \simeq-1 / x^{2}$, the expression for $D(\lambda)$ simplifies essentially and becomes

$$
D(\lambda) \simeq \frac{8 \lambda \varepsilon_{2} d_{0 x}}{\pi\left[2 \varepsilon_{2}\left(\varepsilon_{1}+\varepsilon_{0}\right)+p\left(\varepsilon_{2}-\varepsilon_{1}\right)\left(\varepsilon_{2}-\varepsilon_{0}\right)\right]} .
$$

Such $D(\lambda)$ corresponds to the same dipole potential as $\varphi_{0}^{(x)}$ $\times(\rho, z, \alpha)$ but containing some additional coordinateindependent factor. In other words, the potential outside a nanowire is the potential of some effective dipole $d_{x}=A d_{0 x}$ where the "amplification factor" $A$ characterising the influence of a core-shell structure is equal to

$$
A=\frac{4 \varepsilon_{0} \varepsilon_{2}}{2 \varepsilon_{2}\left(\varepsilon_{1}+\varepsilon_{0}\right)+p\left(\varepsilon_{2}-\varepsilon_{1}\right)\left(\varepsilon_{2}-\varepsilon_{0}\right)} .
$$

At $\varepsilon_{2}=\varepsilon_{1}, p=0$ or $p=1$, Eq. (A4) transforms into the simple formula $A=2 \varepsilon_{0} /\left(\varepsilon_{0}+\varepsilon\right)$ for a uniform cylinder.

The potential of a parallel dipole, $d_{0}=d_{0 z}$, can be found similarly with the only distinction that its potential in a uniform medium is $\varphi_{0}^{(x)}(\rho, z)=d_{0 z} z /\left[\varepsilon_{0}\left(\rho^{2}+z^{2}\right)^{3 / 2}\right]=\operatorname{const}(\alpha)$. Thus we may expect the total potential with the account of image forces to be also $\alpha$-independent and odd in $z$. In this case, the general form of potential is

$$
\varphi^{(z)}(\rho, z)=\left\{\begin{array}{cc}
\frac{d_{0 z} z}{\varepsilon_{1}\left(\rho^{2}+z^{2}\right)^{3 / 2}}+\int_{0}^{\infty} A(\lambda) I_{0}(\lambda \rho) \sin (\lambda z) d \lambda, & \rho<r_{1} ; \\
\int_{0}^{\infty}\left[B(\lambda) I_{0}(\lambda \rho)+C(\lambda) K_{0}(\lambda \rho)\right] \sin (\lambda z) d \lambda, & r_{1}<\rho<r_{2} ; \\
\int_{0}^{\infty} D(\lambda) K_{0}(\lambda \rho) \sin (\lambda z) d \lambda, & \rho>r_{2} .
\end{array}\right.
$$

The matching procedure, similar to Eq. (A2), results in a similar system of linear equations. Its solution for $D(\lambda)$ in the limit of small $\lambda$ is $D(\lambda) \simeq 2 \lambda \varepsilon_{2} d_{0 z} / \pi$ which means that the dipole potential remains unchanged by the core-shell structure, or

$$
A=1 \text {. }
$$

Since our calculations were based on the static Laplace equation, they remain adequate for an oscillating dipole until the corresponding light wavelength remains much larger than the nanowire radius $a$. 
${ }^{1}$ X. Duan and C. M. Lieber, Adv. Mater. (Weinheim, Ger.) 12, 298 (2000).

${ }^{2}$ X. Mei, M. Blumin, M. Sun, D. Kim, Z. H. Wu, H. E. Ruda, and Q. X. Guo, Appl. Phys. Lett. 82, 967 (2003).

${ }^{3}$ A. Shik, J. Appl. Phys. 74, 2951 (1993).

${ }^{4}$ L. D. Landau and E. M. Lifshits, Electrodynamics of Continuous Media (Pergamon, New York, 1984).

${ }^{5}$ M. Bass, Handbook of Optics (McGraw-Hill, New York, 1995), Vol. 2.

${ }^{6}$ P. C. Sercel and K. J. Vahala, Appl. Phys. Lett. 57, 545 (1990).

${ }^{7}$ C. R. McIntyre and L. J. Sham, Phys. Rev. B 45, 9443 (1992).

${ }^{8}$ M. Tsuchiya, J. M. Gaines, R. H. Yan, R. J. Simes, P. O. Holtz, L. A. Coldren, and P. M. Petroff, Phys. Rev. Lett. 62, 466 (1989).

${ }^{9}$ H. Akiyama, T. Someya, and H. Sakaki, Phys. Rev. B 53, R4229 (1996).

${ }^{10}$ F. Vouilloz, D. Y. Oberli, M.-A. Dupertuis, A. Gustafsson, F. Reinhardt, and E. Kapon, Phys. Rev. B 57, 12378 (1998).

${ }^{11}$ J. Wang, M. S. Gudiksen, X. Duan, Y. Cui, and C. M. Lieber, Science 293, 1455 (2001).

${ }^{12}$ J. Qi, A. M. Belcher, and J. M. White, Appl. Phys. Lett. 82, 2616 (2003).

${ }^{13}$ V. Russier and M. P. Pileni, Surf. Sci. 425, 313 (1999).

${ }^{14}$ U. Kreibig and M. Vollmer, Optical Properties of Metal Clusters (Springer, Berlin, 1995).
${ }^{15}$ K. Haraguchi, T. Katsuyama, and K. Hiruma, J. Appl. Phys. 75, 4220 (1994).

${ }^{16}$ P. Lavallard and R. A. Suris, Solid State Commun. 95, 267 (1995).

${ }^{17}$ D. Kovalev, M. Ben Chorin, J. Diener, F. Koch, Al. L. Efros, M. Rosen, N. A. Gippius, and S. G. Tikhodeev, Appl. Phys. Lett. 67, 1585 (1995).

${ }^{18}$ L. J. Lauhon, M. S. Gudiksen, D. Wang, and C. M. Lieber, Nature (London) 420, 57 (2002).

${ }^{19}$ H.-J. Choi, J. C. Johnson, R. He, S.-K. Lee, F. Kim, P. Pauzauskie, J. Goldberger, R. J. Saykally, and P. Yang, J. Phys. Chem. B 107, 8721 (2003).

${ }^{20}$ A. E. Neeves and M. H. Birnboim, J. Opt. Soc. Am. B 6, 787 (1989).

${ }^{21}$ H. S. Zhou, I. Honma, H. Komiyama, and J. W. Haus, Phys. Rev. B 50, 12052 (1994).

${ }^{22}$ S. J. Oldenburg, R. D. Averitt, S. L. Westcott, and N. J. Halas, Chem. Phys. Lett. 288, 243 (1998).

${ }^{23}$ H. E. Ruda and A. Shik, Phys. Rev. B 71, 245328 (2005).

${ }^{24}$ M. Moskovits, Rev. Mod. Phys. 57, 783 (1985).

${ }^{25}$ P. Ils, Ch. Greus, A. Forchel, V. D. Kulakovskii, N. A. Gippius, and S. G. Tikhodeev, Phys. Rev. B 51, 4272 (1995).

${ }^{26}$ I. Webman, J. Jortner, and M. H. Cohen, Phys. Rev. B 15, 5712 (1977). 